\title{
Protective effects of physiological testosterone on advanced glycation end product-induced injury in human endothelial cells
}

\author{
YAPING XIE ${ }^{1}$, DAN YU ${ }^{2}$, JIAHUA WU ${ }^{2}$ and $\mathrm{LIN} \mathrm{LI}^{2}$ \\ ${ }^{1}$ Department of Hematology, Hangzhou No. 1 People's Hospital; ${ }^{2}$ Department of Endocrinology, \\ The Affiliated Sir Run Run Shaw Hospital, School of Medicine, Zhejiang University, Hangzhou, \\ Zhejiang 310016, P.R. China
}

Received August 17, 2015; Accepted August 8, 2016

DOI: $10.3892 / \mathrm{mmr} .2017 .6130$

\begin{abstract}
The effect of testosterone, a sex steroid, on endothelial cells is controversial as it is uncertain if it has a protective effect on them. Whether physiological testosterone can inhibit the deleterious effects of advanced glycation end products (AGEs) on endothelial cells remains to be elucidated. The present study focused on elucidating the effect of testosterone on the injury of endothelial cells induced by AGEs. Human umbilical vein endothelial cells (HUVECs) were cultured in vitro and treated with AGEs in the presence or absence of various concentrations of testosterone. The cell viability in each group was measured using an MTS assay. Early-stage apoptosis was detected using flow cytometry with Annexin V-fluorescein isothiocyanate/propidium iodide double staining, and the expression levels of apoptosis-associated proteins, B cell lymphoma-2 (Bcl-2), Bcl-2-associated X protein (Bax) and caspase-3, were determined using western blot analysis. Oxidative stress and pro-inflammatory parameters in the medium were evaluated using an enzyme-linked immunosorbent assay. The MTS results showed that AGEs significantly decreased the proliferation of HUVECs, whereas a physiological concentration of testosterone alleviated this damage. Physiological concentrations of testosterone protected the HUVECs from AGE-induced apoptosis, mediated by caspase- 3 and $\mathrm{Bax} / \mathrm{Bcl}-2$. In addition, treatment of the HUVECs with AGEs caused a significant decrease in anti-oxidative parameters, but increased the concentrations of malondialdehyde and tumor necrosis factor- $\alpha$. The activation of Janus kinase 2 and signal transducer and activator of transcription 3 was significantly increased by incubation with AGEs. However, pre-incubation with a physiological concentration of testosterone attenuated
\end{abstract}

Correspondence to: Dr Lin Li, Department of Endocrinology, The Affiliated Sir Run Run Shaw Hospital, School of Medicine, Zhejiang University, 3 East Qingchun Road, Hangzhou, Zhejiang 310016, P.R. China

E-mail: lilinzju@126.com

Key words: testosterone, endothelial cells, advanced glycation end products, apoptosis, oxidative stress, tumor necrosis factor- $\alpha$ these changes. Therefore, the data obtained in the present study established the potential role of physiological testosterone in ameliorating AGE-induced damage in HUVECs.

\section{Introduction}

Diabetes mellitus is one of the most prevalent diseases worldwide, and is associated with a number of microvascular complications, including retinopathy, neuropathy and nephropathy, and macrovascular complications, including ischemic heart disease, cerebrovascular disease and peripheral vascular diseases $(1,2)$. Advanced glycation end products (AGEs) have been recognized as an important inducer in diabetes and a number of age-related vasculopathies (3). AGEs are formed by the non-enzymatic glycation reaction of reducing sugars and proteins, nucleic acids and lipids (4). There is increasing evidence demonstrating that AGEs are intimately involved in the pathophysiology of diabetic cardiovascular disease by stimulating oxidative stress, pro-inflammatory effects and apoptotic responses, and modulating vascular stiffness (5-7).

There is a significant difference in atherosclerotic vascular disease between women and men, possibly due to men lacking the protection afforded to women by estrogen (8). For decades, high levels of testosterone have been considered to be detrimental to the cardiovascular system following reports of sudden high-dose anabolic steroid abuse-associated mortality and the higher male incidence of coronary artery diseases $(9,10)$. However, several studies have presented alternative results. In men, as testosterone levels fall with increasing age, the incidence of coronary heart disease increases $(11,12)$. Furthermore, low plasma testosterone levels in men are associated with other known coronary heart disease risk factors, including hypertension, obesity, increased levels of fibrinogen, hyperinsulinemia, diabetes mellitus and adverse lipid profiles (13). However, the effects of the administration of testosterone on atherogenesis are controversial. Testosterone has been reported to increase the extent of atherosclerosis (14), however, its administration has also been reported to cause a decrease in atherosclerosis in low density lipoprotein-receptor knockout mice (15). Similarly, androgens appear to have an anti-atherogenic effect in men (16). Testosterone is the most abundant androgen in males, with a physiological plasma level of $22.7 \pm 4.3 \mathrm{nM}$, and it declines progressively with increasing 
age (17). Previous studies have shown that testosterone at physiological concentrations may have a beneficial effect on the prevention of thrombosis development (18), stimulation of endothelial cell proliferation (19) and activation of endothelial nitric oxide synthase (NOS) (20).

However, whether and how physiological testosterone inhibits the deleterious effects of AGEs on human umbilical vein endothelial cells (HUVECs) remains to be elucidated. Therefore, the present study aimed to investigate the effects of physiological testosterone on AGE-induced injury in HUVECs.

\section{Materials and methods}

Materials. Testosterone, bovine serum albumin (BSA), D-glucose, trypsin/EDTA solution and DMSO were purchased from Sigma-Aldrich; Thermo Fisher Scientific, Inc. (Waltham, MA, USA). The MTS CellTiter 96 Aqueous kit was purchased from Promega (Madison, WI, USA). The Annexin V-fluorescein isothiocyanate (FITC)/propidium iodide (PI) apoptosis detection kit was obtained from BioVision, Inc. (Milpitas, CA, USA). Assay kits for the measurement of NO production, NOS activity, and superoxide dismutase (SOD), glutathione peroxidase (GSH-Px) and malondialdehyde (MDA) concentrations were purchased from Jiancheng Bioengineering Research Institute (Nanjing, China). The tumor necrosis factor (TNF)- $\alpha$ concentration assay kit was obtained from R\&D Systems (Minneapolis, MN, USA).

Preparation of AGE-BSA in vitro. The AGEs were prepared as previously described by $\mathrm{Xu}$ et al (21). Briefly, BSA was incubated under sterile conditions with $50 \mathrm{mM}$ D-glucose in $5 \% \mathrm{CO} 2 / 95 \%$ air at $37^{\circ} \mathrm{C}$ in the dark for 12 weeks. The unincorporated glucose molecules were removed by dialysis overnight against $0.01 \mathrm{M}$ phosphate-buffered saline (PBS). The success of the AGE preparation was determined using a Spectra Max Gemini EM fluorescence reader (Molecular Devices LLC, Sunnyvale, CA, USA). The measurements were performed in triplicate at the excitation wavelength of $370 \mathrm{~nm}$, and the emission peak was observed at $460 \mathrm{~nm}$. The AGEs were stored at $-20^{\circ} \mathrm{C}$ until use. BSA incubated without $\mathrm{D}$-glucose under the same conditions was used as the negative control.

Cell culture. HUVECs were obtained from ScienCell Research Laboratories (Carlsbad, CA, USA). The cells were cultured in endothelial cell growth media with 5\% supplemental fetal bovine serum, 1X endothelial cell growth supplement, $10 \mathrm{U} / \mathrm{ml}$ penicillin and $10 \mu \mathrm{g} / \mathrm{ml}$ streptomycin medium and all components were obtained from ScienCell Research Laboratories) in humidified air, $5 \% \mathrm{CO} 2$ at $37^{\circ} \mathrm{C}$. The cells of the third to fifth passages were used, when specific characteristics of endothelial cells were identified by morphological observation. The HUVECs were separately cultivated at a density of $105 / \mathrm{ml}$ in culture medium containing testosterone at concentrations of $3 \mathrm{nM}, 30 \mathrm{nM}, 3 \mu \mathrm{M}$ and $30 \mu \mathrm{M}$. Following $1 \mathrm{~h}$ of incubation with testosterone, a sample of each cell culture (density $10^{5} / \mathrm{ml}$ ) was treated with $200 \mathrm{mg} / \mathrm{ml}$ AGEs or unmodified BSA. The cell culture was maintained in humidified $5 \% \mathrm{CO}_{2}$ atmosphere at $37^{\circ} \mathrm{C}$. At
$48 \mathrm{~h}$ post-AGE induction, samples from these two culture groups were collected for measurements.

Cell viability analysis. The CellTiter 96 Aqueous kit was used to assess cell viability, according to the manufacturer's protocol, using MTS reagent. Briefly, the cells were plated at a density of $1.5 \times 10^{4}$ cells/well in a 96-well plate and incubated in growth media for $18 \mathrm{~h}$. The cells were further incubated with or without testosterone for $1 \mathrm{~h}$, followed by stimulation with $200 \mu \mathrm{g} / \mathrm{ml}$ of AGEs or unmodified BSA. The cell culture was maintained in humidified $5 \% \mathrm{CO}_{2}$ atmosphere at $37^{\circ} \mathrm{C}$. After $48 \mathrm{~h}$ of culture, MTS solution was added to each well for $3 \mathrm{~h}$, and light absorbance was detected at $490 \mathrm{~nm}$.

Apoptosis assay. To quantify apoptosis, the cells were evaluated by double staining with FITC-conjugated Annexin V and PI, according to the manufacturer's protocol. The cells were washed twice with PBS and stained with Annexin V and PI for $20 \mathrm{~min}$ at room temperature in the dark. The level of apoptosis was determined by measuring the fluorescence of the cells with a FacsCalibur flow cytometer (Becton-Dickinson, San Jose, CA, USA). The viable cells (annexin-V negative, PI negative), cells in early apoptosis (annexin-V positive, PI negative) and cells in late apoptosis or necrosis (annexin-V positive, PI positive) were identified and counted. Data analysis was performed with CellQuest version 3.3 (Becton-Dickinson).

Evaluation of oxidative stress and pro-inflammatory parameters. For the measurement of oxidative stress and pro-inflammatory parameters in the medium, the HUVECs were seeded at density of $10 \%$ well stimulated with BSA $(200 \mu \mathrm{g} / \mathrm{ml})$, AGEs $(200 \mu \mathrm{g} / \mathrm{ml})$, testosterone $(30 \mathrm{nM})$ or AGEs+testosterone $(30 \mathrm{nM})$. Following incubation for $48 \mathrm{~h}$ with humidified $5 \% \mathrm{CO}_{2}$ atmosphere at $37^{\circ} \mathrm{C}$, the culture supernatant was collected. The production of $\mathrm{NO}$, activity of NOS, and concentrations of SOD, GSH-Px and MDA were determined using commercially available assay kits. An enzyme-linked immunosorbent assay (ELISA) for TNF- $\alpha$ was performed on cell culture supernatants using a commercial assay. All procedures were performed according to the manufacturer's protocols.

Western blot analysis. The activation of JAK2 and STAT3 in the HUVECs were assayed using western blot analysis. Following treatment, the cells were extracted using lysis buffer containing $50 \mathrm{mM}$ Tris-Cl, $2.5 \mathrm{mM}$ EGTA, $1 \mathrm{mM}$ EDTA, $10 \mathrm{mM} \mathrm{NaF}, 1 \%$ deoxycorticosterone, $1 \%$ Triton $\mathrm{X}-100$, $1 \mathrm{mM}$ phenyl methylsulfonyl fluoride and $2 \mathrm{mM} \mathrm{Na} 3 \mathrm{VO} 4$. Protein samples from the HUVEC cells were quantified by the bovine serum albumin protein assay kit (Thermo Fisher Scientific, Inc.). The proteins $(30 \mu \mathrm{g})$ were separated using 4-12\% sodium dodecyl sulfate polyacrylamide gel electrophoresis, and were subsequently transferred onto an Immun-Blot PVDF membrane. The membrane was then incubated with primary antibodies overnight at $4^{\circ} \mathrm{C}$. The blots were blocked with $4 \% \mathrm{BSA}$ for $1 \mathrm{~h}$ at room temperature and then probed with the primary antibodies against rabbit primary antibodies [Anti-JAK2 (cat. no. 3230), anti-phosphorylated-JAK2 (cat. no. 3771), anti-STAT3 (cat. no. 8768) and anti-phosphorylated-STAT3 (cat. no. 9145) and anti- $\beta$-actin (cat. no. 4967); 
1:1,000 dilution; Cell Signaling Technology, Inc.] overnight at $4^{\circ} \mathrm{C}$. Following three washes, the blots were subsequently incubated with horseradish peroxidase-labeled anti-rabbit antibody (cat. no. 7074; 1:1,000; Cell Signaling Technology, Inc.) for $1 \mathrm{~h}$ at room temperature. The specific proteins were detected using Super Signal West Pico Chemiluminescent Substrate kit (Thermo Fisher Scientific, Inc.). The band intensities were measured using Quantity One software (Bio-Rad Laboratories, Inc., Hercules, CA, USA) and normalized to total protein.

Statistical analysis. Data are presented as the mean \pm standard error of the mean obtained from three independent experiments. One-way analysis of variance followed by Tukey's HSD post-hoc comparisons were used to determine the differences among multiple groups. SPSS 14.0 software (SPSS, Inc., Chicago, IL, USA) was used. $\mathrm{P}<0.05$ was considered to indicate a statistically significant difference.

\section{Results}

Effect of testosterone on the viability of HUVECs exposed to AGEs. As revealed by the MTS assay, the incubation of HUVECs with AGEs $(200 \mu \mathrm{g} / \mathrm{ml})$ led to a significant reduction in cell proliferation ( $\mathrm{P}<0.05$, vs. control group; Fig. 1). By contrast, in the presence of $30 \mathrm{nM}$ testosterone, a significant increase $(\mathrm{P}<0.05)$ in cell viability was observed, compared with the AGE group, although this effect was not observed at the $3 \mathrm{nM}$ or $3 \mu \mathrm{M}$ concentrations of testosterone. At a supraphysiological concentration $(30 \mu \mathrm{M})$ of testosterone, cell viability was markedly reduced $(\mathrm{P}<0.05)$, compared with the control group and AGE group. Neither the unmodified BSA or DMSO affected cell viability ( $\mathrm{P}>0.05)$, compared with the control group. Collectively, these data demonstrated that the physiological concentration of $30 \mathrm{nM}$ testosterone alleviated AGE-induced cell death.

Effect of testosterone on the apoptosis of HUVECs exposed to AGEs. To evaluate whether the proliferative effect induced by the physiological concentration $(30 \mathrm{nM})$ of testosterone was due to its anti-apoptotic activity, the early-stage apoptotic cells were analyzed using Annexin V/PI double staining. Flow cytometric analysis showed that the apoptotic rate was significantly elevated by AGE treatment, compared with BSA treatment $(\mathrm{P}<0.05$; Fig. $2 \mathrm{~A}-\mathrm{C})$. The effects of testosterone on AGE-induced apoptosis was then examined. Pretreatment of the HUVECs with $30 \mathrm{nM}$ testosterone prevented AGE-induced cytotoxicity, compared with the AGE group $(\mathrm{P}<0.05)$, however, no significant differences were observed at lower or higher testosterone concentrations $(\mathrm{P}>0.05)$, compared with the AGE group (Fig. 2A and D-G). At the supraphysiological concentration of testosterone (Fig. 2F and G), the percentage of apoptotic cells was significantly increased $(\mathrm{P}<0.05$, vs AGE group). These results suggested that the physiological concentration of testosterone protected the HUVECs from AGE-induced apoptosis.

To further examine the molecular mechanisms underlying the anti-apoptotic effect of physiological testosterone, the present study investigated the protein expression levels of caspase-3, Bax and Bcl-2 using western blot analysis. As shown in Fig. $2 \mathrm{H}$ and I, treatment of the HUVECs with

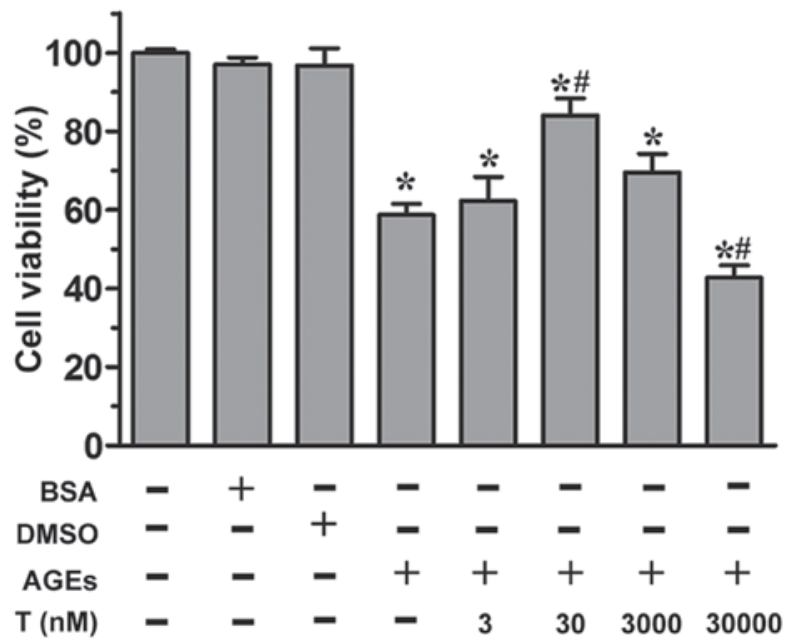

Figure 1. Effect of testosterone on the viability of HUVECs exposed to AGEs. Cell viability was determined using an MTS assay following treatment of the HUVECs with DMSO, unmodified BSA, AGEs, or indicated concentrations of testosterone. Data are shown as the mean \pm standard error of the mean of triplicate experiments. ${ }^{*} \mathrm{P}<0.05$, vs. control; ${ }^{\#} \mathrm{P}<0.05$, vs. AGEs. HUVECs, human umbilical vein endothelial cells; AGEs, advanced glycation end products; BSA, bovine serum albumin; T, testosterone.

AGEs for $48 \mathrm{~h}$ led to a significant increase in the activation of caspase-3 ( $\mathrm{P}<0.05$, vs. BSA group). In addition, the presence of AGEs significantly increased the $\mathrm{Bax} / \mathrm{Bcl}-2$ ratio, compared with that in the BSA group $(\mathrm{P}<0.05$; Fig. $2 \mathrm{H}$ and $\mathbf{J}$ ). Of note, pretreatment of the HUVECs with $30 \mathrm{nM}$ testosterone decreased the activity of caspase-3 and the $\mathrm{Bax} / \mathrm{Bcl}-2$ ratio stimulated by AGEs ( $\mathrm{P}<0.05$, vs. AGE group). However, $3 \mathrm{nM}$ and $3 \mu \mathrm{M}$ testosterone had no effect on the AGE-induced activity of caspase-3 or upregulation of the $\mathrm{Bax} / \mathrm{Bcl}-2$ ratio ( $\mathrm{P}>0.05$, vs. AGE group). Pretreatment with $30 \mu \mathrm{M}$ testosterone led to significant increases in the activation of caspase- 3 and the $\mathrm{Bax} / \mathrm{Bcl}-2$ ratio $(\mathrm{P}<0.05$, vs. AGE group). These data suggested that the protective effect of the physiological concentration $(30 \mathrm{nM})$ of testosterone against AGE-induced apoptosis may be mediated by caspase- 3 and $\mathrm{Bax} / \mathrm{Bcl}-2$.

Effect of physiological testosterone on AGE-induced oxidative stress. A common indication of endothelial cell dysfunction is enhanced oxidation, and AGEs have been associated with this (22). Therefore, the present study investigated the concentrations of MDA and NO, and the activities of SOD, GSH-Px and eNOS to further confirm the protective effects of physiological testosterone on AGE-induced injury in HUVECs. The level of NO (Fig. 3A) and activity of NOS (Fig. 3B) in the HUVECs exposed to AGEs were found to be significantly decreased, compared with those in the BSA group. However, pre-treatment with $30 \mathrm{nM}$ testosterone increased the levels of these indicators $(\mathrm{P}<0.05$, vs. AGE group). Treatment of the HUVECs with AGEs caused a significant decrease in the activities of SOD (Fig. 3C) and GSH-Px (Fig. 3D), compared with the BSA group $(\mathrm{P}<0.05)$. Additionally, pre-incubation with $30 \mathrm{nM}$ testosterone attenuated the changes in the activities of SOD and GSH-Px ( $\mathrm{P}<0.05$, vs. AGE group). As shown in Fig. 3E, the concentration of MDA in the cells treated with AGEs was 
A

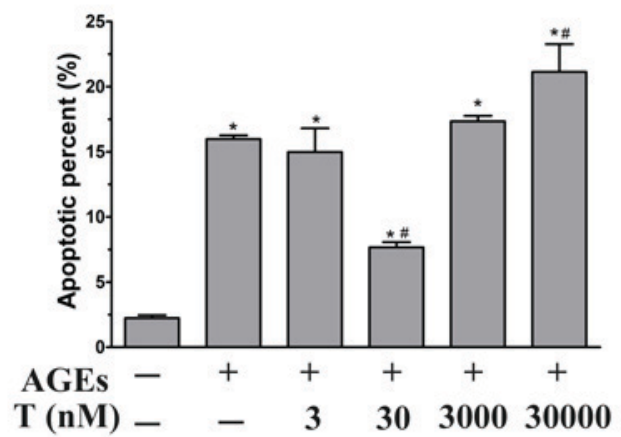

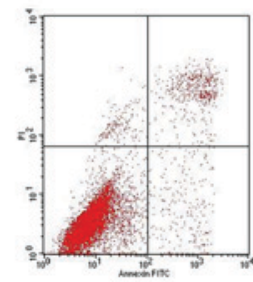

E

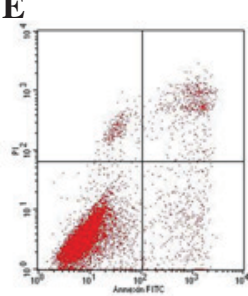

C

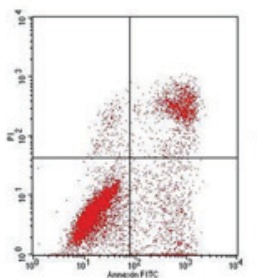

F

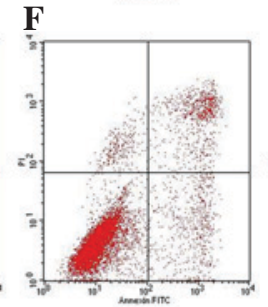

D

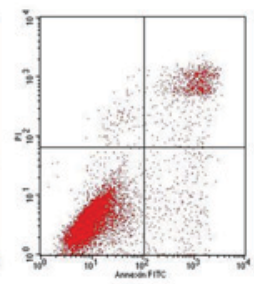

G

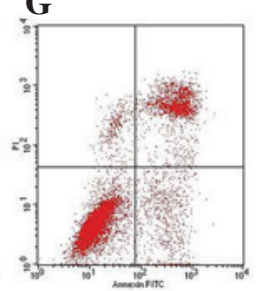

$\mathbf{H}$

I

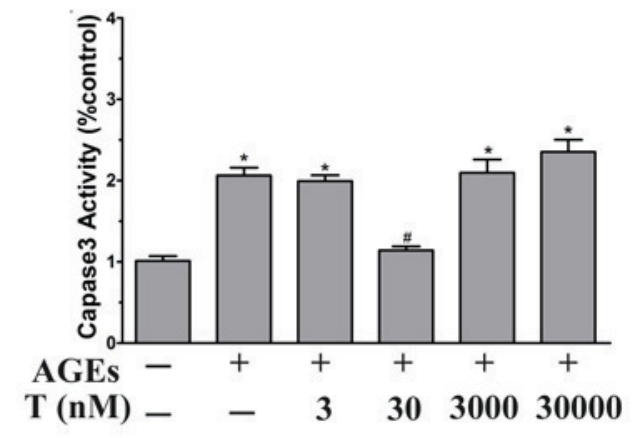

$\mathbf{J}$

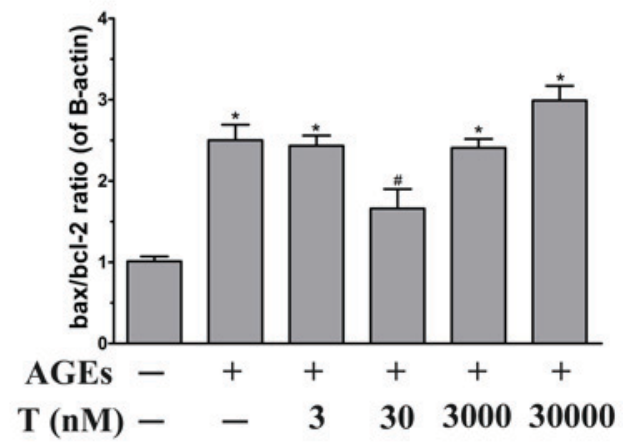

Figure 2. Effect of testosterone on the apoptosis of HUVECs exposed to AGEs. Following stimulation and harvesting of the cells, the levels of apoptosis, vs. necrosis were quantified using AnnexinV-FITC and PI staining with flow cytometric analysis. The representative dot plots are shown. Flow cytometry profiles show Annexin-V-FITC staining on the X-axis and PI on the y-axis. The lower right quadrant represents the percentage of early apoptotic cells in each condition. (A) Apoptosis was evaluated following treatment of the HUVECs with (B) unmodified BSA, (C) AGEs or (D) 3 nM, (E) 30 nM, (F) $3 \mu$ M or (G) $30 \mu \mathrm{M}$ testosterone. (H) Representative western blot images and quantitative immunoblot analysis of (I) cleaved caspase3 normalized to total caspase3 and the $(\mathrm{J}) \mathrm{Bax} / \mathrm{Bcl}-2$ ratio, normalized to $\beta$-actin. Data are shown as the mean \pm standard error of the mean of triplicate experiments. ${ }^{*} \mathrm{P}<0.05$, vs. $\mathrm{BSA}$; ${ }^{*} \mathrm{P}<0.05$, vs. AGEs. HUVECs, human umbilical vein endothelial cells; AGEs, advanced glycation end products; FITC, fluorescein isothiocyanate; PI, propidium iodide; BSA, bovine serum albumin; T, testosterone; Bcl-2, B cell lymphoma-2; Bax, Bcl-2-associated X protein.

significantly higher, compared with that in the BSA group $(\mathrm{P}<0.05)$. By contrast, there was a significant decrease in the concentration of MDA when the HUVECs were pre-treated with $30 \mathrm{nM}$ testosterone $(\mathrm{P}<0.05$, vs. AGE group). In the presence of $30 \mathrm{nM}$ testosterone without AGEs, none of the parameters were significantly different, compared with those of the BSA group. Collectively, these results suggested that a physiological concentration $(30 \mathrm{nM})$ of testosterone may act as an anti-oxidative agent by protecting HUVECs against AGE-induced oxidative stress.

Effect of physiological testosterone on the AGE-induced secretion of TNF- $\alpha$. Endothelial cells are producers of and targets for cytokines. It has been established that AGEs can stimulate the production of TNF- $\alpha$ in HUVECs (23). In order to investigate whether a physiological concentration $(30 \mathrm{nM})$ of testosterone can suppress the AGE-induced secretion of TNF- $\alpha$, the levels of TNF- $\alpha$ were measured in the media of cultured HUVECs using an ELISA kit. Following the application of AGEs for $48 \mathrm{~h}$, the level of TNF- $\alpha$ increased significantly, compared with that of the BSA group, as shown in Fig. 3F $(\mathrm{P}<0.05)$, whereas the level of TNF- $\alpha$ was markedly suppressed by treatment with $30 \mathrm{nM}$ testosterone ( $\mathrm{P}<0.05$, vs. AGE group). JAK2/STAT3 pathway activation. AGE-induced endothelial 

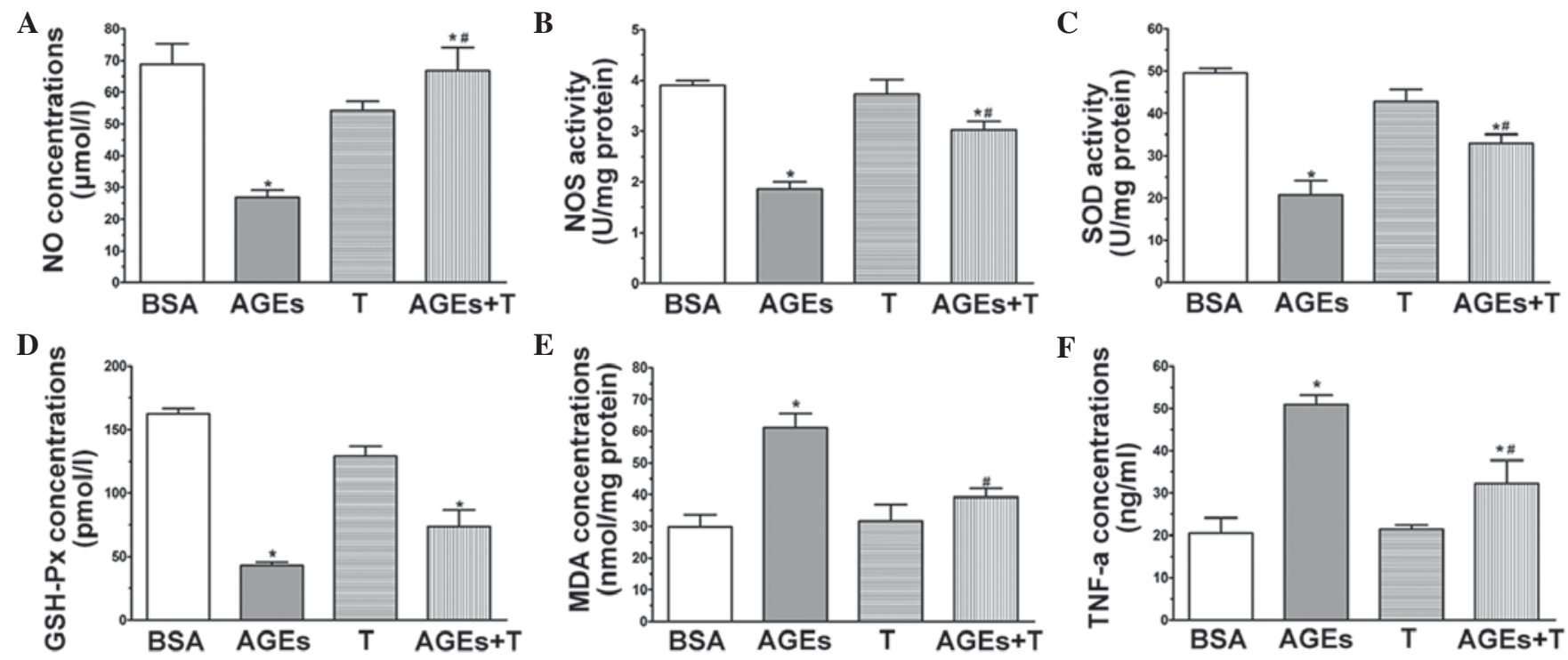

Figure 3. Physiological testosterone suppresses AGE-induced oxidative stress and secretion of TNF- $\alpha$ in human umbilical vein endothelial cells. The images show quantitative assessments of (A) NO concentration, (B) NOS activity, (C) SOD activity, (D) GSH-Px concentration, (E) MDA concentration and (F) TNF- $\alpha$ concentration were performed using specific ELISA following $48 \mathrm{~h}$ exposure to advanced glycation end products (AGEs) with or without $30 \mathrm{nM}$ testosterone. Data are shown as the mean \pm standard error of the mean of triplicate experiments. ${ }^{*} \mathrm{P}<0.05$, vs. BSA; ${ }^{"} \mathrm{P}<0.05$, vs. AGEs. NO, nitric oxide; NOS, nitric oxide synthase; SOD, superoxide dismutase; MDA, malondialdehyde; GSH-Px, glutathione peroxidase; TNF- $\alpha$, tumor necrosis factors- $\alpha$; AGEs, advanced glycation end product; BSA, bovine serum albumin; T, testosterone.
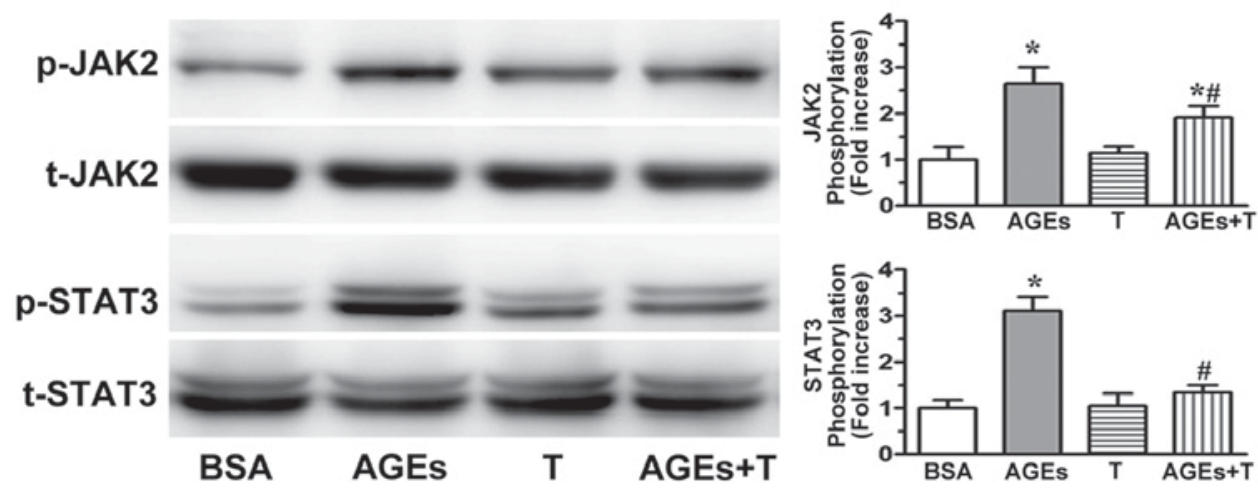

Figure 4. Physiological testosterone inhibits AGE-induced JAK2/STAT3 pathway activation. Representative western blot images and quantification of p-JAK2 and p-STAT3 in human umbilical vein endothelial cells following $48 \mathrm{~h}$ exposure to AGEs with or without $30 \mathrm{nM}$ testosterone and normalized against total protein. Unmodified BSA, AGEs or $30 \mathrm{nM}$ testosterone. Data are shown as the mean \pm standard error of the mean of triplicate experiments. " $\mathrm{P}<0.05$, vs. $\mathrm{BSA}$; ${ }^{\text {"P }} \mathrm{P}<0.05$, vs. AGEs. AGEs, advanced glycation end product; JAK2, Janus kinase 2; STAT3, signal transducer and activator of transcription 3; p-phosphorylated; $\mathrm{t}-$, total; BSA, bovine serum albumin; T, testosterone.

dysfunction has been significantly correlated with JAK2/STAT3 activation $(24,25)$. In the present study, western blot analysis was used to examine whether physiological testosterone ameliorated the AGE-induced JAK2/STAT3 pathway activation. As shown in Fig. 4, the activation of JAK2 and STAT3 were significantly increased following incubation with AGEs. However, this process was reduced by pre-incubating the cells with $30 \mathrm{nM}$ testosterone. These results indicated that the physiological concentration of $30 \mathrm{nM}$ testosterone may attenuate AGE-induced endothelial dysfunction via the JAK2/STAT3 pathway.

\section{Discussion}

The most important characteristics linked to diabetic vascular complications include high levels of circulating glucose, increased oxidative stress and the accumulation of AGEs (26). Various studies have demonstrated that AGEs are pivotal in several diabetes-associated vasculopathies $(27,28)$. In the present study, it was established that AGEs caused apoptosis in HUVECs, induced the expression of oxidative stress and pro-inflammatory markers and activated JAK2/STAT3 signaling. The present study revealed that a physiological concentration of testosterone alleviated AGE-induced apoptosis, oxidative stress and inflammation in the HUVECs. In addition, the results showed that testosterone at a supraphysiological concentration increased the injury induced by AGEs.

It is widely reported that AGEs induce apoptosis significantly in HUVECs $(7,29)$. However, whether testosterone can inhibit this AGE-induced apoptosis remains to be elucidated. In the present in vitro study, the results of the MTS assay showed that $30 \mathrm{nM}$ testosterone effectively attenuated the 
reduction in cell viability induced by AGEs. By contrast, at higher concentrations, testosterone had a dose-dependent cytotoxic effect on the endothelial cells. As the MTS assay does not discriminate between necrosis and apoptosis, Annexin V/PI analysis was performed using flow cytometry. The pretreatment of HUVECs with $30 \mathrm{nM}$ testosterone led to a marked reduction in the number of apoptotic cells. However, $30 \mu \mathrm{M}$ testosterone promoted the apoptosis induced by AGEs. Testosterone at physiological plasma levels of $22.7 \pm 4.3 \mathrm{nM}$ exhibit progressive age-related decline, whereas the incidence of coronary vascular diseases has been observed to increase with age (18). Previous studies have supported the beneficial effects of physiological testosterone on endothelial cell function in vivo and in vitro $(17,18)$. Animal studies have shown that the replacement of testosterone prevents aortic cholesterol accumulation in cholesterol-fed, orchidectomized male rabbits (30) and in low-density lipoprotein receptor knockout mice (15). Furthermore, the replacement of physiological testosterone inhibits fatty streak formation in the testicular feminized mouse (31). In a previous in vitro study by Jin et al (18), physiological concentrations of testosterone were suggested to have a beneficial effect on the hemostatic system through enhancement of anticoagulant activity resulting from stimulating the expression of tissue factor pathway inhibitor and tissue plasminogen activator, and inhibiting the secretion of plasminogen activator inhibitor-1 by the endothelium. The present study also aimed to elucidate the mechanisms underlying the inhibition of apoptosis by testosterone. The AGE-induced apoptosis involved the activation of caspase-3, which appeared to be important for the progression of apoptotic cell death. Additionally, the present study revealed that the ratio of Bax, an apoptosis promoter, to $\mathrm{Bcl}-2$, an apoptosis inhibitor, was markedly upregulated in endothelial cells stimulated by AGEs. The present study also demonstrated that $30 \mathrm{nM}$ testosterone significantly decreased the Bax/Bcl-2 ratio and attenuated the AGE-induced activation of caspase-3.

A variety of factors, including reactive oxygen species (ROS), lipid oxidation enzymes and inflammatory cytokines, can result in vascular endothelial cell damage (32). ROS affects lipids and leads to lipid peroxidation, which consequently produces MDA. MDA may combine with proteins, amino acids and other cellular components, changing the structure of phospholipids. However, the antioxidants, SOD and GSH-Px, may also be involved, in which SOD may cooperate with GSH-Px to remove free radicals (33). In the present study, AGEs significantly decreased the activities of SOD and GSH-Px, but increased the contents of MDA. Following pre-treatment with $3 \mathrm{nM}$ testosterone, the HUVECs were observed to resist damage from AGEs, reduce the content of MDA, and increase the activities of SOD and GSH-Px. NOS, one of the key active enzymes in maintaining the physiological function of endothelial cells, showed the capacity to remove free radicals in vivo. In addition, NOS is an enzyme involved in the formation of NO, which is in turn responsible for vasodilatation, blood pressure regulation, cardiac contractility, and the mediation of immunity during bacterial infections and inflammation (34). Compared with the control group, the expression levels of NO and NOS in the AGE group were found to be lower. However, their expression levels increased following pre-treatment with $30 \mathrm{nM}$ testosterone. These results suggested that the ameliorating effect of physiological testosterone on AGE-induced apoptosis occurs partly through the regulation of the anti-oxidation and NO pathway. AGEs can also alter vessel wall homeostasis in a pro-atherogenic manner through the release of inflammatory cytokines (5). As reported in a previous study (23), the secretion of TNF- $\alpha$ was significantly enhanced following incubation with AGEs in the HUVECs. However, $30 \mathrm{nM}$ of testosterone was more effective in protecting the cells against the increase in TNF- $\alpha$ induced by AGEs.

JAK2 and STAT3 are factors known to transduce signals initiated by several growth factors and cytokines. The activated JAK2 tyrosine-phosphorylate and the latent cytoplasmic STAT3 are involved in AGE-induced cell damage (35). In the present study, it was found that the activation of JAK2 and STAT3 were associated with the AGEs. In addition, physiological testosterone significantly inhibited the activation of JAK2 and STAT3. These results suggested that inhibition of JAK2/STAT3 signaling activity may be useful for alleviating AGE-induced cell damage.

In conclusion, the results of the present study showed that AGEs induced endothelial cell apoptosis in vitro and that this change was prevented by pretreatment with physiological testosterone. It was suggested that downregulating the $\mathrm{Bax} / \mathrm{Bcl}-2$ ratio and inhibiting the activation of caspase-3 by physiological testosterone may protect the HUVECs from AGE-induced apoptosis. Physiological concentrations of testosterone were also found to suppress oxidative stress, the expression of inflammatory cytokines and the activation of JAK2/STAT3 induced by AGEs. These findings demonstrated the beneficial effect of physiological testosterone on AGE-induced damage in HUVECs.

\section{Acknowledgements}

This study was supported by the Natural Science Foundation of Zhejiang Province of China (grant no. LQ14H070001) and the Administration of Traditional Chinese Medicine of Zhejiang Province (grant no. 2015ZA058).

\section{References}

1. Rahman S, Rahman T, Ismail AA and Rashid AR: Diabetes-associated macrovasculopathy: Pathophysiology and pathogenesis. Diabetes Obes Metab 9: 767-780, 2007.

2. Cooper ME, Bonnet F, Oldfield M and Jandeleit-Dahm K: Mechanisms of diabetic vasculopathy: An overview. Am J Hypertens 14: 475-486, 2001.

3. Neves D: Advanced glycation end-products: A common pathway in diabetes and age-related erectile dysfunction. Free Radic Res 47 Suppl 1: 49-69, 2013.

4. Prasad A, Bekker P and Tsimikas S: Advanced glycation end products and diabetic cardiovascular disease. Cardiol Rev 20: 177-183, 2012.

5. Basta G, Schmidt AM and De Caterina R: Advanced glycation end products and vascular inflammation: Implications for accelerated atherosclerosis in diabetes. Cardiovasc Res 63: 582-592, 2004.

6. Goldin A, Beckman JA, Schmidt AM and Creager MA: Advanced glycation end products: Sparking the development of diabetic vascular injury. Circulation 114: 597-605, 2006.

7. Min C, Kang E, Yu SH, Shinn SH and Kim YS: Advanced glycation end products induce apoptosis and procoagulant activity in cultured human umbilical vein endothelial cells. Diabetes Res Clin Pract 46: 197-202, 1999. 
8. Mendelsohn ME and Karas RH: The protective effects of estrogen on the cardiovascular system. N Engl J Med 340: 1801-1811, 1999.

9. Bagatell CJ and Bremner WJ: Androgens in men-uses and abuses. N Engl J Med 334: 707-714, 1996.

10. Sullivan ML, Martinez CM, Gennis P and Gallagher EJ: The cardiac toxicity of anabolic steroids. Prog Cardiovasc Dis 41 $1-15,1998$.

11. Jones RD, Nettleship JE, Kapoor D, Jones HT and Channer KS Testosterone and atherosclerosis in aging men: Purported association and clinical implications. Am J Cardiovasc Drugs 5: 141-154, 2005.

12. Choi BG and McLaughlin MA: Why men's hearts break: Cardiovascular effects of sex steroids. Endocrinol Metab Clin North Am 36: 365-377, 2007.

13. Smith AM, English KM, Malkin CJ, Jones RD, Jones TH and Channer KS: Testosterone does not adversely affect fibrinogen or tissue plasminogen activator (tPA) and plasminogen activator inhibitor-1 (PAI-1) levels in 46 men with chronic stable angina. Eur J Endocrinol 152: 285-291, 2005.

14. Adams MR, Williams JK and Kaplan JR: Effects of androgens on coronary artery atherosclerosis and atherosclerosis-related impairment of vascular responsiveness. Arterioscler Thromb Vasc Biol 15: 562-570, 1995

15. Nathan L, Shi W, Dinh H, Mukherjee TK, Wang X, Lusis AJ and Chaudhuri G: Testosterone inhibits early atherogenesis by conversion to estradiol: Critical role of aromatase. Proc Nat Acad Sci USA 98: 3589-3593, 2001

16. English KM, Mandour O, Steeds RP, Diver MJ, Jones TH and Channer KS: Men with coronary artery disease have lower levels of androgens than men with normal coronary angiograms. Eur Heart J 21: 890-894, 2000.

17. Jin H, Lin J, Fu L, Mei YF, Peng G, Tan X, Wang DM, Wang W and Li YG: Physiological testosterone stimulates tissue plasminogen activator and tissue factor pathway inhibitor and inhibits plasminogen activator inhibitor type 1 release in endothelial cells. Biochem Cell Biol 85: 246-251, 2007.

18. Jin H, Wang DY, Mei YF, Qiu WB, Zhou Y, Wang DM, Tan XR and Li YG: Mitogen-activated protein kinases pathway is involved in physiological testosterone-induced tissue factor pathway inhibitor expression in endothelial cells. Blood Coagul Fibrinolysis 21: 420-424, 2010.

19. Cai J, Hong Y, Weng C, Tan C, Imperato-McGinley J and Zhu YS: Androgen stimulates endothelial cell proliferation via an androgen receptor/VEGF/cyclin A-mediated mechanism. Am J Physiol Heart Circ Physiol 300: H1210-H1221, 2011.

20. Yu J, Akishita M, Eto M, Ogawa S, Son BK, Kato S, Ouchi Y and Okabe T: Androgen receptor-dependent activation of endothelial nitric oxide synthase in vascular endothelial cells: Role of phosphatidylinositol 3-kinase/akt pathway. Endocrinology 151: $1822-1828,2010$

21. Xu Y, Feng L, Wang S, Zhu Q, Zheng Z, Xiang P, He B and Tang D: Calycosin protects HUVECs from advanced glycation end products-induced macrophage infiltration. J Ethnopharmacol 137: 359-370, 2011.
22. Zhuang X, Pang X, Zhang W, Wu W, Zhao J, Yang H and Qu W: Effects of zinc and manganese on advanced glycation end products (AGEs) formation and AGEs-mediated endothelial cell dysfunction. Life Sci 90: 131-139, 2012.

23. Rashid G, Benchetrit S, Fishman D and Bernheim J: Effect of advanced glycation end-products on gene expression and synthesis of TNF-alpha and endothelial nitric oxide synthase by endothelial cells. Kidney Int 66: 1099-1106, 2004.

24. Grimm S, Ott C, Horlacher M, Weber D, Hohn A and Grune T: Advanced-glycation-end-product-induced formation of immunoproteasomes: Involvement of RAGE and Jak2/STAT1. Biochem J 448: 127-139, 2012.

25. Nedić O, Rattan SI, Grune T and Trougakos IP: Molecular effects of advanced glycation end products on cell signalling pathways, ageing and pathophysiology. Free Radic Res 47 (Suppl 1): S28-S38, 2013

26. Rodiño-Janeiro BK, González-Peteiro M, Ucieda-Somoza R, González-Juanatey JR and Alvarez E: Glycated albumin, a precursor of advanced glycation end-products, up-regulates NADPH oxidase and enhances oxidative stress in human endothelial cells: Molecular correlate of diabetic vasculopathy. Diabetes Metab Res Rev 26: 550-558, 2010.

27. Orasanu G and Plutzky J: The pathologic continuum of diabetic vascular disease. J Am Coll Cardiol 53 (Suppl 5): S35-S42, 2009.

28. Méndez JD, Xie J, Aguilar-Hernández M and Méndez-Valenzuela V: Trends in advanced glycation end products research in diabetes mellitus and its complications. Mol Cell Biochem 341: 33-41, 2010.

29. Sang HQ, Gu JF, Yuan JR, Zhang MH, Jia XB and Feng L: The protective effect of Smilax glabra extract on advanced glycation end products-induced endothelial dysfunction in HUVECs via RAGE-ERK1/2-NF- $\mathrm{B}$ pathway. J Ethnopharmacol 155: 785-795, 2014

30. Alexandersen P, Haarbo J, Byrjalsen I, Lawaetz $\mathrm{H}$ and Christiansen C: Natural androgens inhibit male atherosclerosis: A study in castrated, cholesterol-fed rabbits. Circ Res 84: 813-819, 1999.

31. Nettleship JE, Jones TH, Channer KS and Jones RD: Physiological testosterone replacement therapy attenuates fatty streak formation and improves high-density lipoprotein cholesterol in the Tfm mouse: An effect that is independent of the classic androgen receptor. Circulation 116: 2427-2434, 2007.

32. Hulsmans M and Holvoet P: The vicious circle between oxidative stress and inflammation in atherosclerosis. J Cell Mol Med 14: 70-78, 2010

33. Ho E, Karimi Galougahi K, Liu CC, Bhindi R and Figtree GA: Biological markers of oxidative stress: Applications to cardiovascular research and practice. Redox Biol 1: 483-491, 2013.

34. Allen JD, Giordano T and Kevil CG: Nitrite and nitric oxide metabolism in peripheral artery disease. Nitric Oxide 26: 217-222, 2012.

35. Huang JS, Guh JY, Hung WC, Yang ML, Lai YH, Chen HC and Chuang LY: Role of the Janus kinase (JAK)/signal transducters and activators of transcription (STAT) cascade in advanced glycation end-product-induced cellular mitogenesis in NRK-49F cells. Biochem J 342: 231-238, 1999. 